Polymer Journal, Vol. 38, No. 7, pp. 694-702 (2006)

(C) 2006 The Society of Polymer Science, Japan

\title{
Conversion and Separation Pattern of Lignocellulosic Carbohydrates through the Phase-separation System
}

\author{
Keigo MiKame and Masamitsu FunAOKA ${ }^{\dagger}$ \\ Faculty of Bioresources, Mie University, 1577 Kurima-machiya, Tsu 514-8507, Japan
}

(Received September 26, 2005; Accepted March 7, 2006; Published June 9, 2006)

\begin{abstract}
Through the phase-separation reaction system with sulfuric acid, lignocellulosics which form an interpenetrating polymer network within the cell wall were separated almost quantitatively into different phases within $20 \mathrm{~min}$. The yield of carbohydrate of acid layer after the $60 \mathrm{~min}$ phase-separation treatment was $71 \%$ of raw material (98\% of carbohydrate). A total yield of original lignin without grafting cresol in lignocresol, acid phase sugar and cresol phase sugar was $96 \%$ of raw material at the 60 min phase-separation treatment. It was possible to control conversion pattern of lignocellulosics component with the quantity of the phenol derivatives. [doi:10.1295/polymj.PJ2005138] KEY WORDS Lignin / Carbohydrate / Phase-separation System / Lignophenol /
\end{abstract}

In recent years, lignocellulosic materials have shown interesting features to be used as raw materials for industrial material production. Lignocellulosics are renewable, available in abundance and inexpensive, offering great potential for transformation into chemical feedstocks or fuels. ${ }^{1,2}$ However, the lignocellulosics must be separated into individual components before use as chemical feedstocks. Separating lignocellulosic components is extremely difficult, since the lignocellulosic have the interpenetrating polymer network structure composed of lignin, hemicellulose and cellulose. ${ }^{3}$ So far the lignocellulosics have been treated with strong alkaline for paper making or hydrolyzed with strong acid for pretreatment of ethanol fermentation. ${ }^{4-6}$ Lignins are subjected to very complicated modifications and deactivated during these treatments, ${ }^{7-9}$ thus denatured lignin have been treated as if a waste.

Recently a new process for separating lignocellulosics into lignin and carbohydrate moieties with the conversion to highly reactive forms at room temperature was developed by Funaoka et al. ${ }^{10}$ This process includes a phase-separative reaction system composed of phenol derivatives and concentrated acid which are immiscible with each other. In the process the concentrated acid is not only a solvent for carbohydrate, but also works as a catalyst for the fragmentation and phenolation of lignin, whereas phenol derivatives act as phenolation agents, a barrier to minimize the attack of acid on the lignin and a solvent for the lignin fractions (Figure 1). The separated lignin derivatives (lignophenol) have several unique functions, which conventional lignins do not have, in spite of retention of the original interunit linkages: these include highly phenolic property, no conjugated system, light color compara- ble with native lignin, solid-liquid transformation and high immobilization capability for proteins (enzyme). ${ }^{11,12}$ Furthermore, the molecular structures of lignophenol can be secondarily controlled using a nucleophilic attack of phenolic units to adjacent carbons, resulting in a change of the important functionalities such as phenolic activity and molecular weight. These original functions are due to the selective hybridization of monomeric phenol derivatives at $\mathrm{C}-1$ positions, leading to liner type structures composed mainly of 1,1-bis(aryl)propane-type units. On the other hand, the carbohydrates were hydrolyzed to monomeric, oligomeric and polymeric forms during the phase-separation treatment. Recently the acid hydrolysis of lignocellulosic materials has been attracted again because of energy issues, global warming caused by greenhouse gases, raw material of biodegradable polymer and pharmaceutical supplies from hemicellulose.

In this study, conversion and separation pattern of plant cell wall carbohydrate through the phase-separation system was investigated. The composition and molecular weight distribution of separated carbohydrates and material balance of lignocellulosic components were examined with various condition of phaseseparation treatment. The conversion and separation pattern of lignin component was reported in previous papers. ${ }^{10-12}$

\section{EXPERIMENTAL}

\section{Lignocellulosic Materials}

Air dried Yezo spruce (Picea jezoensis) wood were ground successively in a Wiley mill and a ultra centrifugal mill to pass an 80 mesh screen, and extracted with ethanol-benzene $(1: 2, \mathrm{v} / \mathrm{v})$ for $48 \mathrm{~h}$.

${ }^{\dagger}$ To whom correspondence should be addressed (Tel: +81-59-231-9521, Fax: +81-59-231-9521, E-mail: funaoka@ bio.mie-u.ac.jp). 


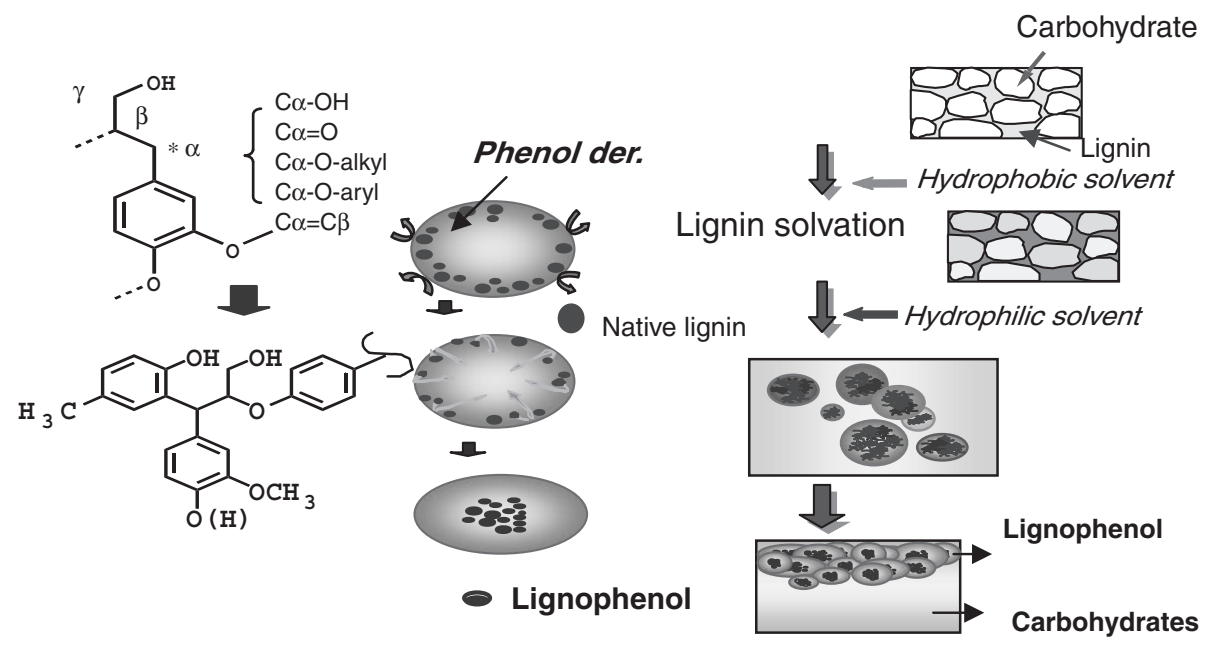

Figure 1. Phase-separation system (1step process) for conversion and separation of lignocellulosic components.

\section{Phase-separation Treatment 1step Process}

$p$-Cresol $(10 \mathrm{~mL} / \mathrm{g}$ wood) was added to spruce wood meals with stirring at room temperature. After $10 \mathrm{~min}, 72 \%$ sulfuric acid $(15 \mathrm{~mL} / \mathrm{g}$ wood) was added to the mixture and the vigorous stirring was continued at $30^{\circ} \mathrm{C}$ for the prescribed time.

The reaction mixture was rapidly separated into the organic and aqueous phases by centrifugation. The organic phase was taken up and added dropwise to an excess amount of ethyl ether with vigorous stirring. The precipitates were dissolved in acetone and insoluble materials were removed by centrifugation. The acetone solution was then concentrated under reduced pressure and added dropwise to an excess amount of ethyl ether with stirring. The precipitated lignin derivative (lignocresol) was collected by centrifugation.

\section{Phase-separation Treatment 2step Process}

For solvation of lignin with phenol derivatives, $3 \mathrm{~mol} / \mathrm{C} 9$ of $p$-cresol dissolved in acetone was added to wood meal and acetone was evaporated with stirring. Sulfuric acid $(72 \% 15 \mathrm{~mL} / \mathrm{g}$ wood) was added to the mixture and the vigorous stirring was continued at $30^{\circ} \mathrm{C}$ for the prescribed time. Ninety seconds before a reaction end time, $5 \mathrm{~mL}$ of $p$-cresol was added to reaction mixture. After stirring for $90 \mathrm{~s}$, the reaction mixture was rapidly separated into the organic and aqueous phases by centrifugation. Extraction and purification of lignocresol from organic phase was preformed as well as 1step process.

\section{Molecular Weight Distribution of Lignocresol}

Gel permeation chromatogram of lignocresol was determined on a JASCO PU-1580 High-Performance Liquid Chromatograph (HPLC).

(GPC condition)
Column; Shodex GPC KF-801, 802, 803, 804 $(8 \mathrm{~mm} \mathrm{ID} \times 300 \mathrm{~mm})$,

Eluent; THF

Flow rate; $1.0 \mathrm{~mL} / \mathrm{min}$

Temp.; $40^{\circ} \mathrm{C}$

Detect; $280 \mathrm{~nm}$

Standard: polystyrene standard

\section{Contents of Grafted Cresol in Lignocresol}

The amount of cresol combined in lignophenols was calculated based on the signal intensity of cresolic methyl protons against aromatic protons of $p$-nitrobenzaldehyde (internal standard) on a ${ }^{1} \mathrm{H}$ NMR spectrum.

\section{Carbohydrate Composition}

The aqueous phase after phase-separation treatment was diluted to $3 \%$ sulfuric acid concentration with distilled water, and was boiled for $4 \mathrm{~h}$. After cooling, ribose as an internal standard was added. The solution was neutralized and desalted by barium hydroxide, and was analyzed by the SHIMADZU HPLC Reducing Sugar Analysis System.

(HPLC condition)

Column: Shim-pack ISA-07/S2504 (4 mm ID. $\times 25$ cm L.)

Eluent: A; $0.1 \mathrm{M}$ potassium borate buffer $(\mathrm{pH} 8.0)$, B; $0.4 \mathrm{M}$ potassium borate buffer $(\mathrm{pH} 9.0)$, Flow rate: $0.6 \mathrm{~mL} / \mathrm{min}$

Gradient: A $100 \%$ to B $100 \%(2 \% / \mathrm{min})$

Temp: $65^{\circ} \mathrm{C}$

Reaction reagent; $1 \% \mathrm{~L}$-arginine, $3 \%$ Boric acid, 0.5 $\mathrm{mL} / \mathrm{min}$

Reaction temp.; $150^{\circ} \mathrm{C}$, detection wave; Ex-320 nm, Em-430 nm

The composition of carbohydrate in the organic phase was analyzed as follows. The organic phase 


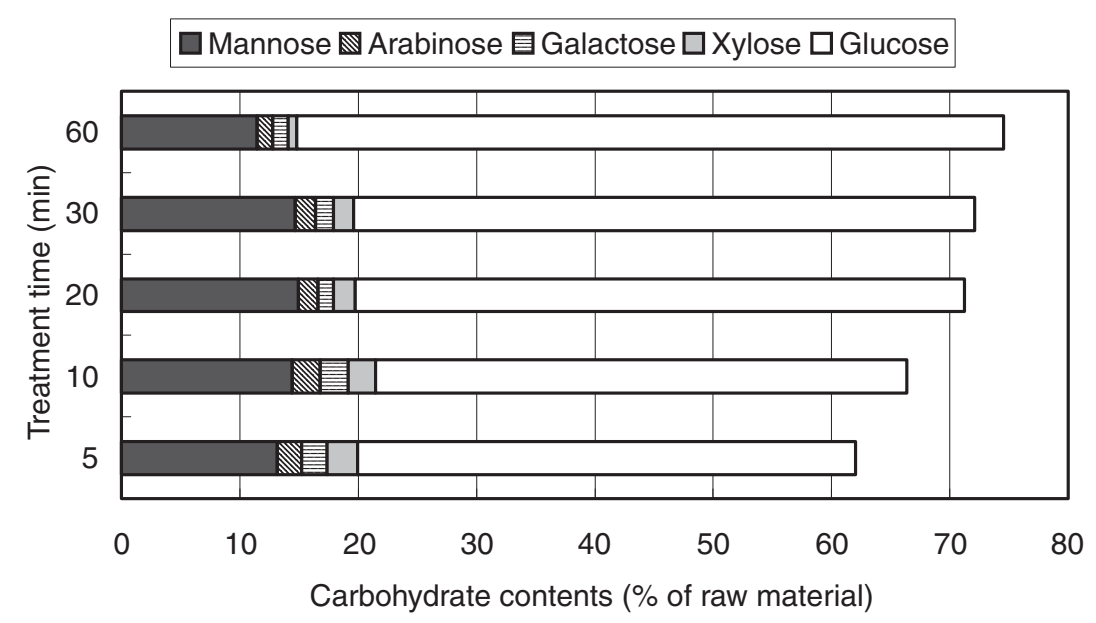

Figure 2. Carbohydrate compositions of the sulfuric acid phase after the 1step phase-separation treatment.

was added to diethyl ether with stirring. The precipitates were dissolved in acetone and the insoluble materials were collected by centrifugation. The insoluble materials $(10 \mathrm{mg})$ were treated with $1 \mathrm{~mL}$ of $72 \%$ sulfuric acid at $30^{\circ} \mathrm{C}$ for $1 \mathrm{~h}$. The reaction mixture was diluted to $3 \%$ sulfuric acid concentration with distilled water and heated in an autoclave at $120^{\circ} \mathrm{C}$ for $1 \mathrm{~h}$. After cooling, ribose as an internal standard was added. After neutralization and desalting, the supernatant was analyzed by the same method as the aqueous phase.

\section{Molecular Weight Distribution of Carbohydrates}

The aqueous phase after sulfuric acid hydrolysis was neutralized and desalted by barium hydroxide. The solution was analyzed by gel permeation chromatography (GPC).

(GPC condition)

Column: Asahipak GS-620HQ, GS-520HQ, GS$320 \mathrm{HQ}$, GS-220HQ (7.6 mm ID. $\times 30 \mathrm{~cm} \mathrm{L.)}$

Eluent: $\mathrm{H}_{2} \mathrm{O}$

Flow rate: $0.6 \mathrm{~mL} / \mathrm{min}$

Temp: $50{ }^{\circ} \mathrm{C}$

Detect: RI

Standard: Pullulan, maltose, maltotriose, maltopentaose, maltoheptaose

\section{RESULTS AND DISCUSSION}

\section{Carbohydrate Composition}

The wood fibers are composed of cellulose containing crystalline region coated with noncrystalline hemicellulose, and hydrophobic lignin, forming semi interpenetrating polymer network structure. A part of lignin is linked to hemicellulose through ether or ester linkages. For complete hydrolysis, cellulose must be swollen by treatment with a concentrated acid such as more than $65 \%$ of sulfuric acid or more than $85 \%$ of phosphoric acid. However concentrated acid treatment causes self-condensation of lignin, which limits dissolution of carbohydrate (the belt effect). Consequently, it takes $2-4 \mathrm{~h}$ to dissolve cellulose. On the other hand, in the phase-separation system lignin was solvated with phenol derivative to prevent from the attack of concentrated acid, self-condensation of lignin (belt effect) were suppressed. Thus dissolution of cellulose is accelerated.

Figure 2 shows the carbohydrate composition of the aqueous phase after the 1step phase separation treatment. With phase separation treatment for $5 \mathrm{~min}$, the content of hemicellulose sugar (mannose, arabinose, galactose, xylose) was about $20 \%$ of raw material, corresponding to $31 \%$ of total neutral sugar in the sulfuric acid phase. However, the content of hemicellulose sugar in the aqueous phase was about $19 \%$ of total neutral sugar after $60 \mathrm{~min}$. This reason is that noncrystalline hemicellulose is preferentially hydrolyzed at the early step of phase-separation treatment, followed by hydrolysis of cellulose. Total neutral sugar from the sulfuric acid phase after the phase separation treatment was $62 \%$ of raw material ( $86 \%$ of total carbohydrate) in 5 min treatment time, and the neutral sugar in the sulfuric acid phase increased with the treatment, the content was $71 \%$ of raw material (98\% of total carbohydrate) in 20 min treatment and the content was $75 \%$ (104\% of total carbohydrate) in 60 min treatment.

Figure 3 shows the carbohydrate composition of the aqueous phase after the 2step phase separation treatment. Total neutral sugar from the sulfuric acid phase after the 2step phase separation treatment was less than the 1step phase separation treatment; the yields were $65 \%$ of raw material (90\% of total carbohydrate) in 20 min treatment and the content was 68\% (95\% of total carbohydrate) in $60 \mathrm{~min}$ treatment.

Because the $p$-cresol works not only as conversion 


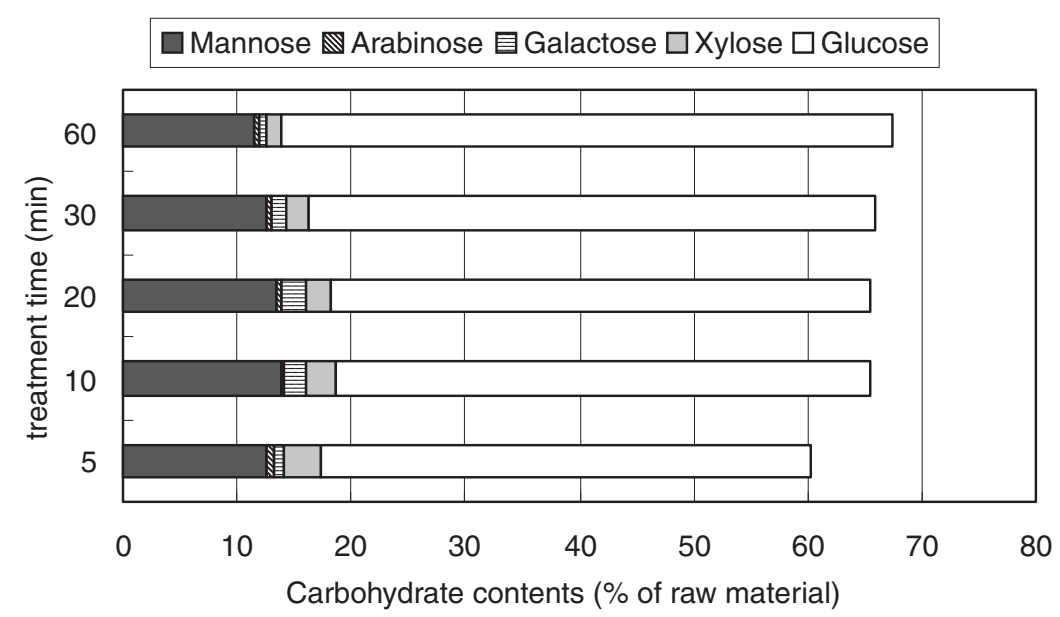

Figure 3. Carbohydrate compositions of the sulfuric acid phase after the 2step phase-separation treatment.

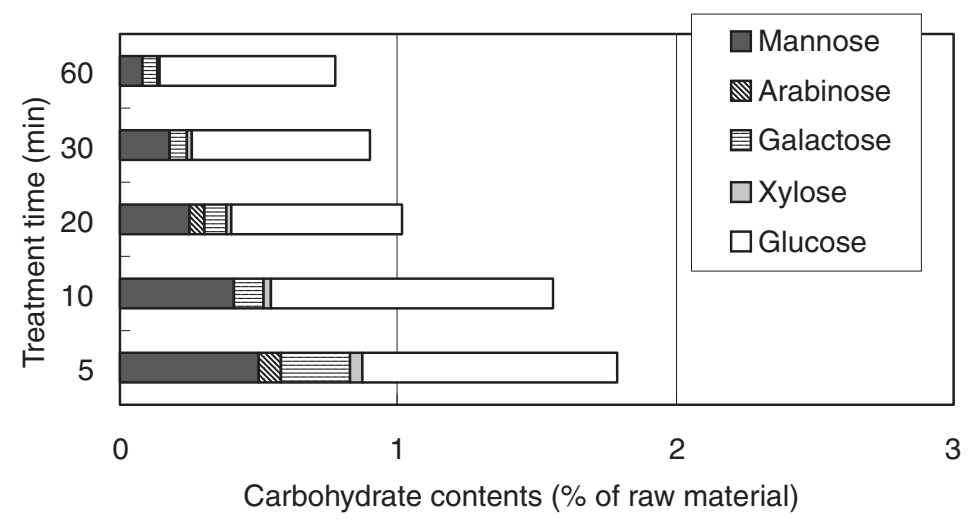

Figure 4. Carbohydrate compositions of the cresolic phase after the 1step phase-separation treatment.

agent for lignin, but also as solvents for extracting the resulting lignocresol, the lignocresol is efficiently separated from lignocellulosics. Thus the belt effect was inhibited and hydrolysis of cellulose was accelerated.

On the other hand, the content of carbohydrate of the cresolic phase was getting decreased with the treatment time; $1.8 \%$ of raw material at $5 \mathrm{~min}$ and $0.7 \%$ of raw material at $60 \mathrm{~min}$ of the 1 step phase separation treatment (Figure 4). The content of hemicellulose sugar was $40 \%$ of total neutral sugar in the cresolic phase at $5 \mathrm{~min}$ of the phase separation treatment. Because a part of lignin is linked to hemicellulose, the hemicellulose was separated into the cresolic phase with lignocresol at an early stage of phase separation treatment. However, the content of hemicellulose sugar was decreased to $18 \%$ after 60 min treatment, because the linkages between lignin and hemicellulose were cleaved through the contact with acid at the interface between two phases and hemicellulose sugar was transported to the aqueous phase. The content of carbohydrate of the cresolic phase after the 2step phase separation treatment was less than the 1step phase separation treatment; $0.15 \%$ of raw material at
60 min treatment (Figure 5). The ratio of hemicellulose sugar in cresolic phase carbohydrates after the 2 step phase separation treatment was higher than the 1step phase separation treatment.

Because the transferable carbohydrate to cresolic phase is difficult for low content of phenol derivatives, and the carbohydrate which can shift on this condition was restricted almost exclusively to affinity carbohydrate to lignin for example lignin-carbohydrate complex (LCC).

\section{Molecular Weight Distribution of Carbohydrates}

The carbohydrates in the sulfuric acid phase were consisted of high molecular weight fractions (more than $\left.M_{\mathrm{w}}=100,000\right)$ and low molecular weight fraction (less than $M_{\mathrm{w}}=2,000$ ) (Figures 6 and 7). The high molecular weight fraction was $65 \%$ of the acid phase carbohydrate after the 10 min 1step phaseseparation treatment. The high molecular weight fraction was decreased to $24 \%$ of the acid phase carbohydrate after the $240 \mathrm{~min}$ 1step phase-separation treatment. Analysis of carbohydrate composition and the ${ }^{1} \mathrm{H}$ NMR spectrum suggested that this fraction is 


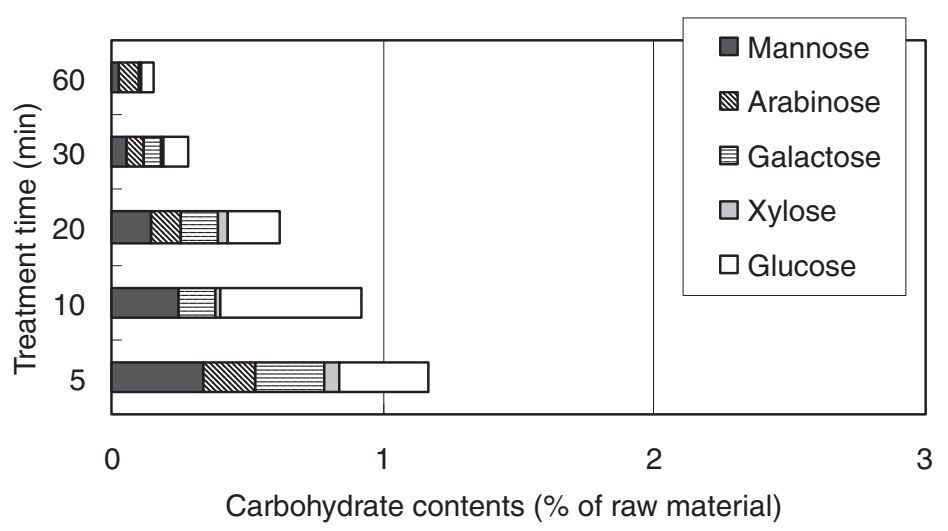

Figure 5. Carbohydrate compositions of the cresolic phase after the 2 step phase-separation treatment.

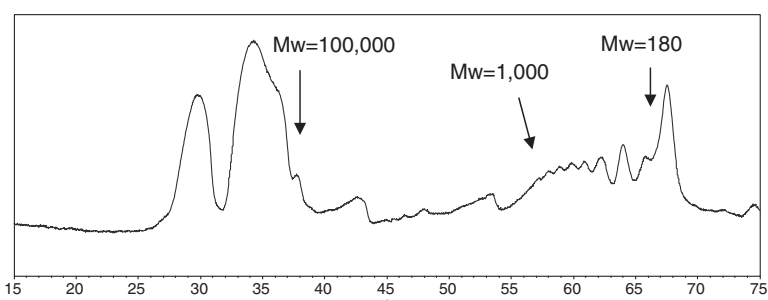

1step phase-separation treatment time 5 min

Retention time (min)

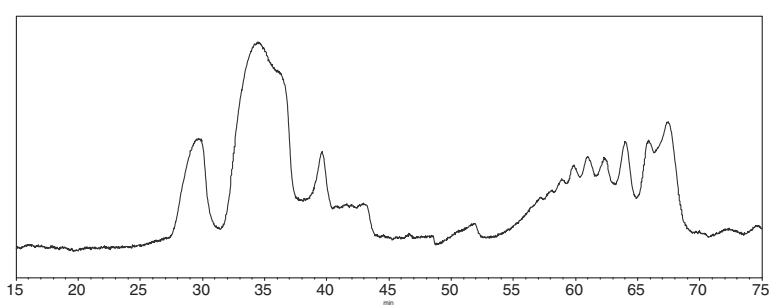

1step phase-separation treatment time $10 \mathrm{~min}$

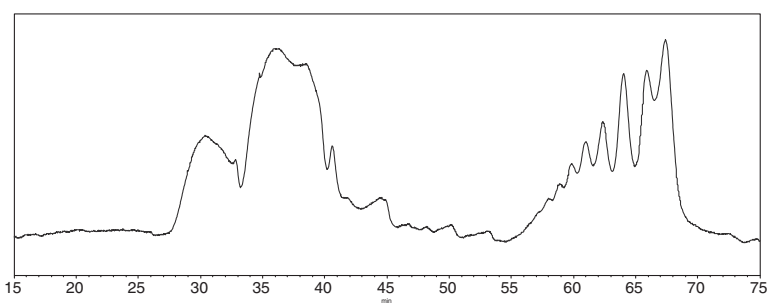

1step phase-separation treatment time $20 \mathrm{~min}$

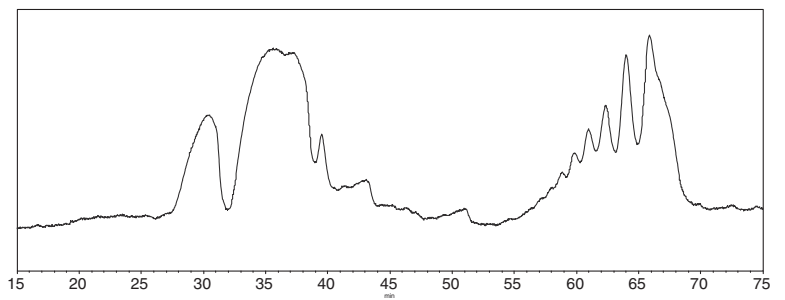

1step phase-separation treatment time $30 \mathrm{~min}$

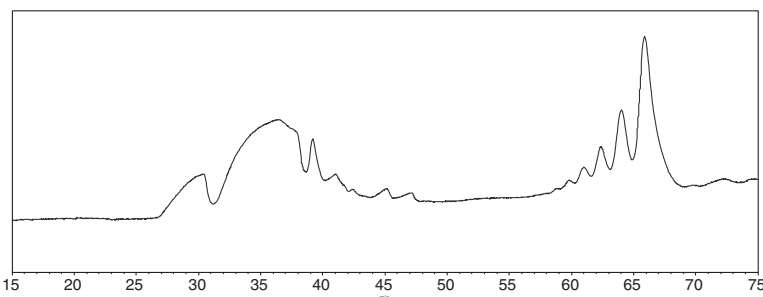

1step phase-separation treatment time $60 \mathrm{~min}$

Figure 6. GPC profiles of carbohydrates in the acid phase after 1step phase-separation treatment. 


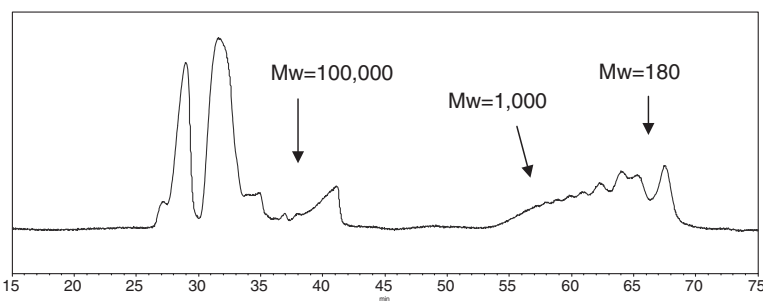

Retention time (min)
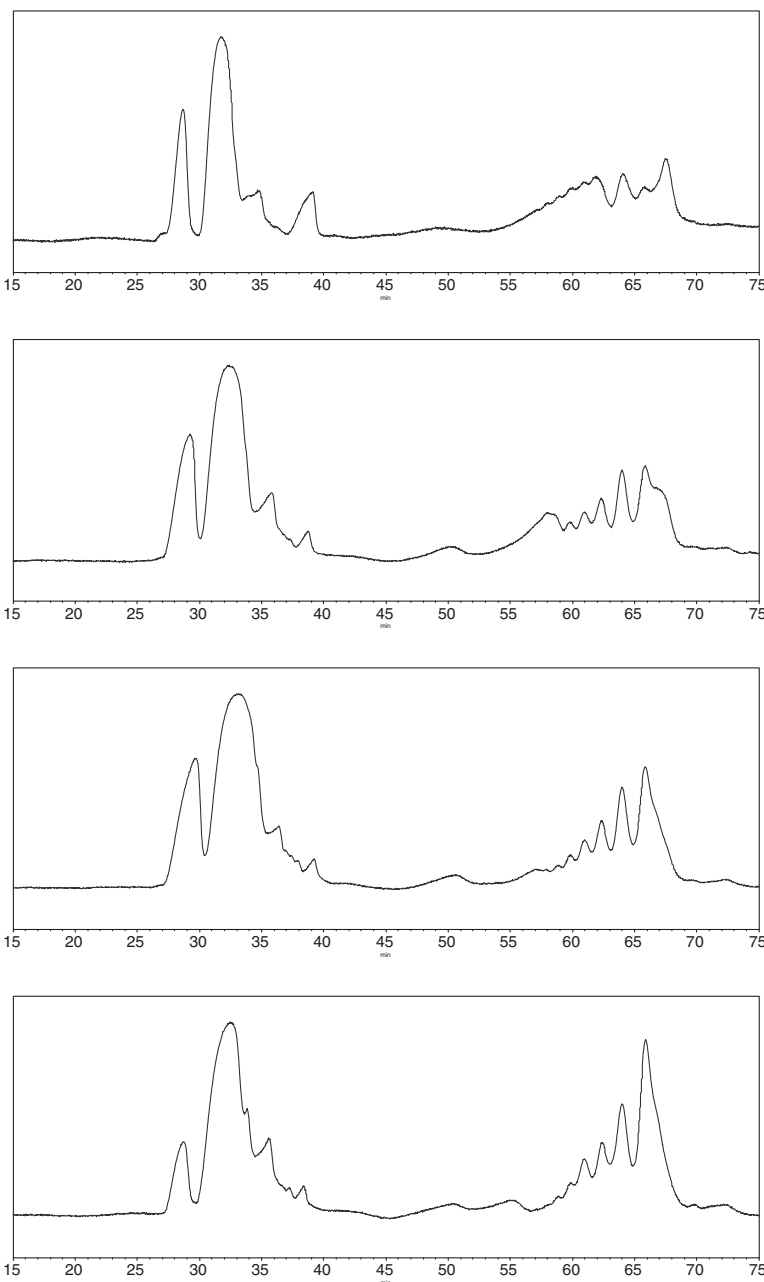

2step phase-separation

treatment time $5 \mathrm{~min}$

2step phase-separation

treatment time $10 \mathrm{~min}$

2step phase-separation

treatment time $20 \mathrm{~min}$

2step phase-separation

treatment time $30 \mathrm{~min}$

2step phase-separation

treatment time $60 \mathrm{~min}$

Figure 7. GPC profiles of carbohydrates in the acid phase after 2step phase-separation treatment.

water-soluble polymers containing glucose as major carbohydrate component, because the spectrum do not have aromatic ring signals $(6-8 \mathrm{ppm})$ and presented many broad signals for carbohydrate (3-5 ppm) (Figure 8). ${ }^{13}$ On the other hands the low molecular weight fraction consisted of mixtures of the oligosaccharides with various molecular sizes. A part of oligosaccharides was hydrolyzed to monosaccharides with extended reaction time.

In the 2step phase-separation treatment, the carbohydrates in the sulfuric acid phase were higher molecular weight than the 1step phase-separation treatment. This difference of hydrolysis rate is based on the extraction effect of lignophenol from lignocellulosic with phenol derivatives.

\section{Characterization of Lignocresol}

In order to investigate total material balance and characterization of lignocresol, lignocresol was purified from cresolic phase after the phase separation treatment. In the 1step process the yield of lignocresol increased with the treatment time, the yield was $21.4 \%$ of raw material at 5 min treatment and $29.8 \%$ of raw material at 60 min treatment (Figure 9). In order to calculate the amount of original lignin, the amount of cresol combined with lignin was determined by ${ }^{1} \mathrm{H}$ NMR. The amount of grafted cresol increased from $0.79 \mathrm{~mol} / \mathrm{C} 9$ to $0.85 \mathrm{~mol} / \mathrm{C} 9$ slightly by extending treatment time from $5 \mathrm{~min}$ to $60 \mathrm{~min}$ (Figure 11). The molecular weight of lignocresol decreased to $M_{\mathrm{w}}=9,400$ at $60 \mathrm{~min}$ from $M_{\mathrm{w}}=34,000$ 


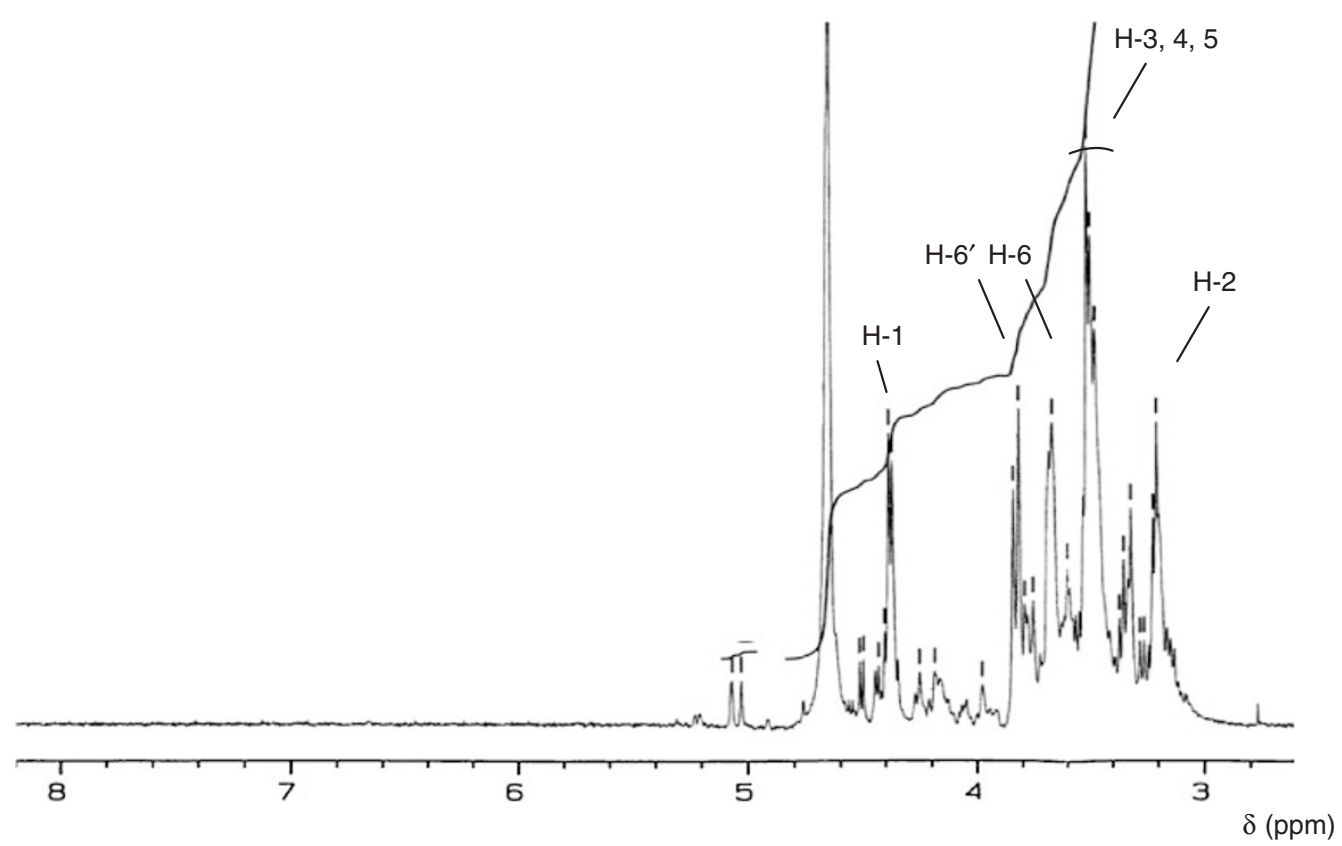

Figure 8. ${ }^{1} \mathrm{H}$ NMR spectrum of high molecular weight fraction from the acid phase after 1step phase-separation treatment for 30 min.

1step process

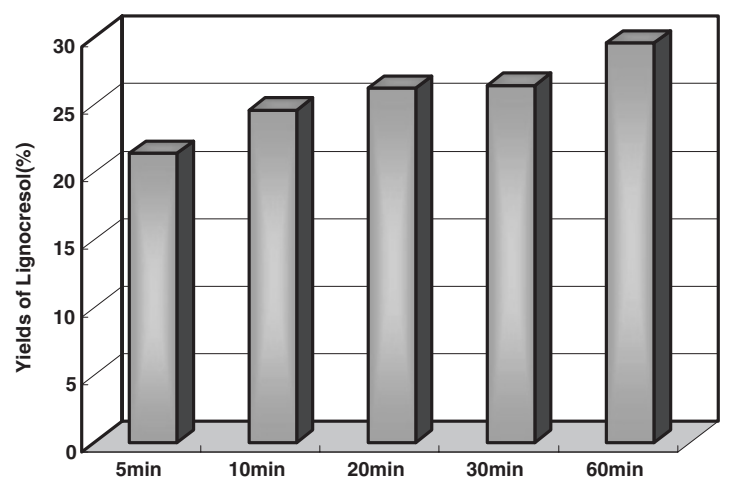

2step process

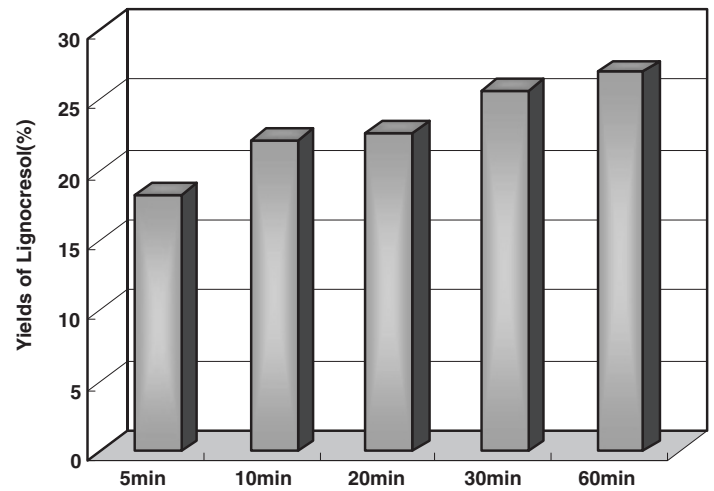

Figure 9. Relationship between the time of phase-separation treatment and the yields of lignocresol.
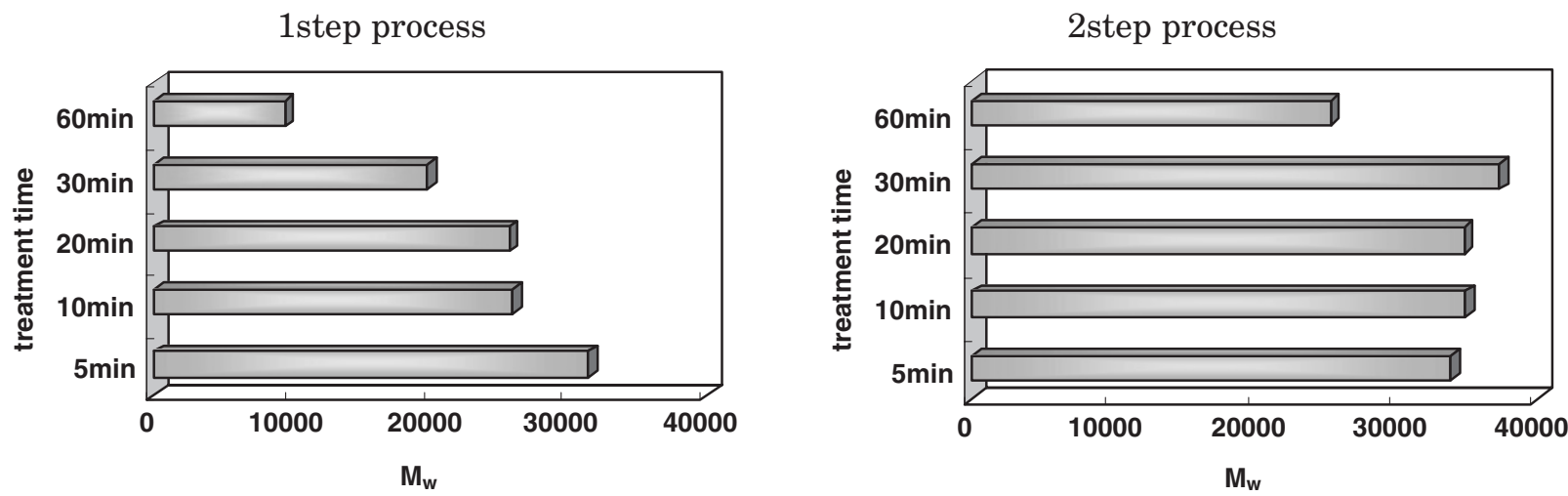

Figure 10. Relationship between the time of phase-separation treatment and the molecular weight of lignocresol.

at 5 min treatment with the increase in grafted cresol (Figure 10). These results showed that depolymerization of lignin by cleavage of benzyl aryl ether proceed with the treatment and $p$-cresol is grafted at the same time.

On the other hand in the 2step process, the yield of 
1step process

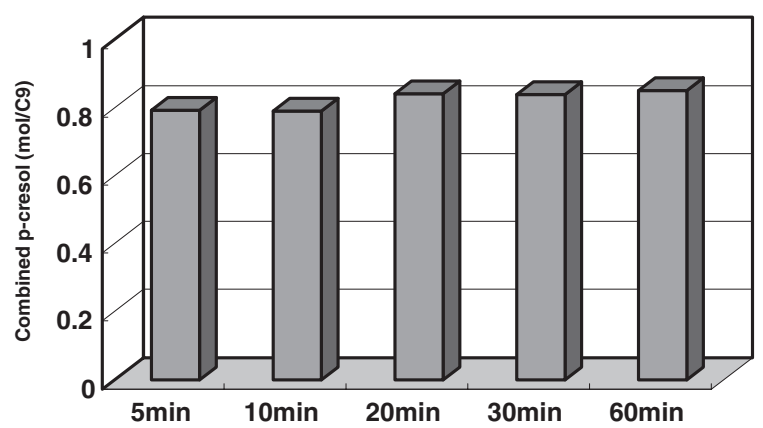

2step process

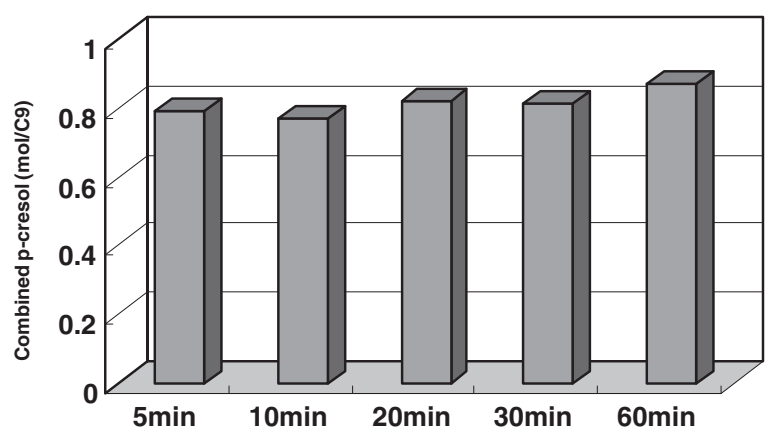

Figure 11. Relationship between the time of phase-separation treatment and the contents of cresol combined with lignin.

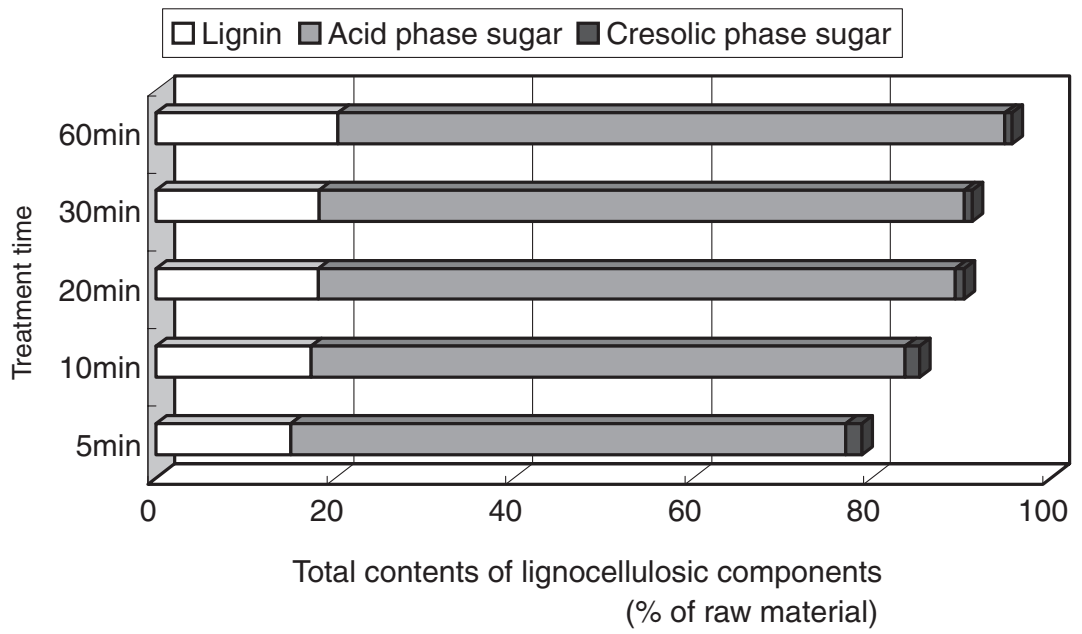

Figure 12. Total material balance of lignocellulosic components in 1step phase-separation treatment.

lignocresol was lower than the 1step process. The molecular weights of lignocresol from 2 step process were higher than 1step process. These results show that the separation rate of lignocellulosics in the 1step phase separation system is higher than the 2step phase separation system.

\section{CONCLUSIONS}

In the phase-separation reaction system composed of phenol derivatives and concentrated acid, lignin and carbohydrates, which form an interpenetrating polymer network within the cell wall, were separated almost quantitatively into different phases within $20 \mathrm{~min}$ (Figure 12). A total yield of lignocresol (cresol free) and sugars from the acid phase and the cresol phase was $96 \%$ of raw material at the 60 min 1 step phase-separation treatment. In the 2 step process, the total yield was $88 \%$ of raw material at the $60 \mathrm{~min}$ (Figure 13).

In this system, because lignin was solvated with phenol derivative to prevent from the attack of concentrated acid, self-condensation of lignin was sup- pressed. Thus the belt effect of lignin to restrict carbohydrates hydrolysis was released, so that carbohydrates were rapidly swollen and dissolved in the concentrated acid. In this acid hydrolysis of carbohydrates, the hydrolysis of noncrystalline hemicellulose was preceded and a part of hemicellulose was separated into the cresolic phase with lignophenol at an early stage of phase separation treatment. With extended reaction time, the carbohydrate content in the organic phase got much lower because of the decomposition of lignin-carbohydrate complex.

Separated carbohydrates were consisted of various sizes of oligosaccharide and high molecular weight water-soluble glucose polymers. The water-soluble polysaccharide and oligosaccharide can be use for thickening agent etc. and functional food, respectively. These carbohydrate components can be converted readily to simple sugars by dilute acid hydrolysis. The monosaccharides also convert to ethanol or lactic acid through fermentation. The monosaccharides from hemicellulose are expected as pharmaceuticals, for example xylitol. 


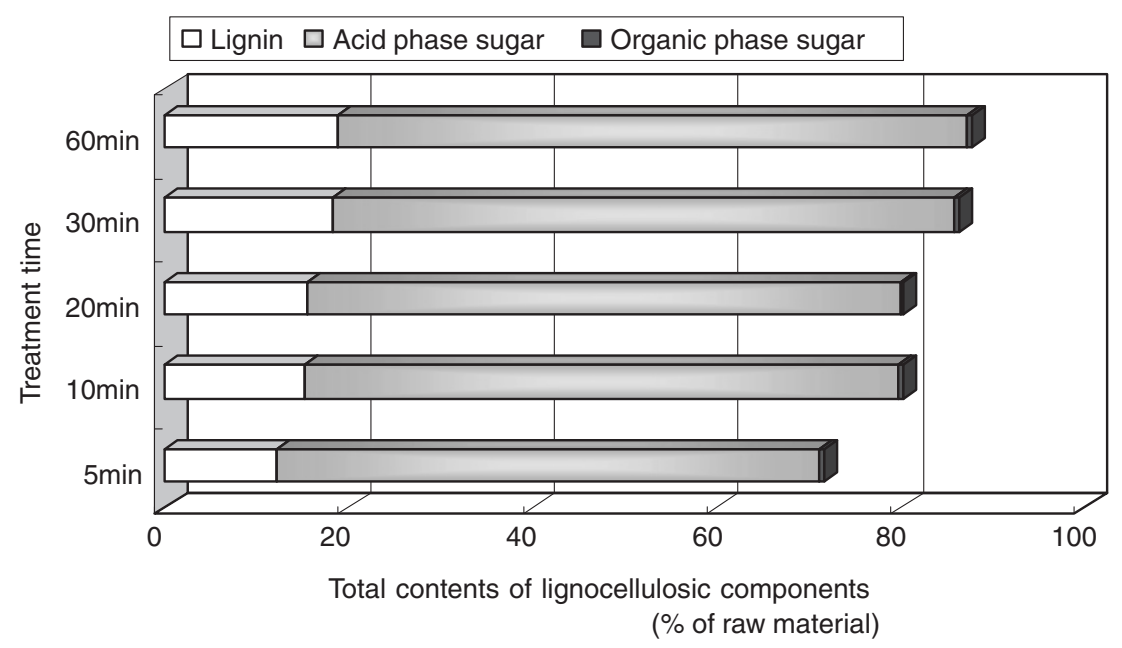

Figure 13. Total material balance of lignocellulosic components in 2step phase-separation treatment.

\section{REFERENCES}

1. I. S. Goldstein, For. Prod. J., 31, 63 (1981).

2. F. Parisi, Adv. Biochem. Eng. Biotechnol., 38, 53 (1989).

3. E. S. Lipinsky, in "Hydrolysis of Cellulose: Mechanisms of Enzymatic and Acid Catalysis" L. Jurasek and R. D. Brown, Jr., Ed., 1979, p 1.

4. I. S. Goldstein, Tappi J., 63, 141 (1980).

5. Y. S. Cheng, Bioresour. Technol., 83, 1 (2002).

6. J. D. McMikkan, in "Pretreatment of lignocellulosics biomass" M. E. Himmel, J. O. Barker, and R. P. Overrend,
Ed., 1994, p 292.

7. B. Johansson and G. E. Miksche, Acta Chem. Scand., 23, 924 (1969).

8. J. M. Harkin, Adv. Chem. Ser., 59, 65 (1966).

9. S. Yasuda, N. Terashima, and T. Ito, Mokuzai Gakkaishi, 26, 552 (1980).

10. M. Funaoka and I. Abe, Tappi J., 72, 145 (1989).

11. M. Funaoka and S. Fukatsu, Holzforschung, 50, 245 (1996).

12. M. Funaoka, Polym. Int., 47, 277 (1998).

13. L. A. Fluggue, J. T. Blank, and P. A. Petillo, J. Am. Chem. Soc., 121, 7228 (1999). 